Check for updates

Cite this: RSC Adv., 2017, 7, 37522

Received 4th May 2017

Accepted 6th July 2017

DOI: 10.1039/c7ra05039a

rsc.li/rsc-advances

\section{Comparative bone regeneration study of hardystonite and hydroxyapatite as filler in critical- sized defect of rat calvaria $\uparrow$}

\author{
Kunal Khanna, ${ }^{a}$ Amit Jaiswal, ${ }^{\mathrm{b}}$ Rohit V. Dhumal, ${ }^{\mathrm{c}}$ Nilakash Selkar, ${ }^{\mathrm{c}}$ Pradip Chaudhari, \\ Vivek P. Soni, ${ }^{e}$ Geeta R. Vanage ${ }^{c}$ and Jayesh Bellare (iD *afg
}

There is a very significant and well-known clinical need for the development of new osteoinductive materials and the establishment of alternative therapies for the treatment of bone tissue loss or failure resulting from injury or disease as the transplantation of tissues in patients with these injury or disease is severely limited by donor scarcity and is highly associated to the risk of immune rejection and disease transfer. Herein, we studied in vivo bone response by quantifying efficacy and safety of three scaffold variations: (1) nanofibrous polycaprolactone (PCL), (2) PCL-hydroxyapatite (HA), and (3) PCLhardystonite (HS) against SHAM as the control. Diffraction pattern from TEM showed that native HA and HS were polycrystalline and they leached higher ppm of calcium, phosphorus and zinc as compared to $\mathrm{PCL}-\mathrm{HA}$ and $\mathrm{PCL}-\mathrm{HS}$ in which HA, HS were incorporated in PCL nanofibers. The study was performed on $8 \mathrm{~mm}$ critical-sized rat calvarial defects analyzed at two timepoints, 6 and 12 weeks. The bone regenerated by $\mathrm{PCL}-\mathrm{HS}$ promoted higher growth than that by SHAM and PCL alone at 12 weeks with comparable bone mineral density in all groups at both time points. PCL-HS showed potential for bone growth similar to that for PCL-HA. Histology data showed dense bone interface being formed at the site in both the PCL-HS and PCL-HA groups. Therefore, HS was found to have comparable functionality with commercial HA. No significant differences were noted in any of the blood parameters but there were differences in serum biochemistry parameters of triglyceride and creatine levels among groups, which are indirectly related to bone forming potential and directly to safety of kidney function, while the other parameters were unchanged and within the normal range. Thus, we conclude that the HS material can be a suitable substitute for bone tissue engineering.

\section{Introduction}

A number of methods have been employed to fabricate scaffolds for bone tissue engineering and a variety of bone regeneration studies conducted by several groups have shown the potential of

${ }^{a}$ Center for Research in Nanotechnology and Science, Indian Institute of Technology Bombay, Mumbai, India

${ }^{b}$ Centre for Biomaterials, Cellular and Molecular Theranostics, VIT University Vellore, Tamil Nadu, India

'National Centre for Preclinical Reproductive and Genetic Toxicology, National Institute for Research in Reproductive Health, J. M. Street, Parel, Mumbai 400012, India

${ }^{d}$ Division of Animal Oncology, Advanced Centre for Treatment, Research \& Education in Cancer, Navi Mumbai, India

${ }^{e}$ Department of Biosciences and Bioengineering, India

${ }^{f}$ Department of Chemical Engineering, Indian Institute of Technology Bombay, Powai, Mumbai - 400076, India. E-mail: jb@iitb.ac.in; Tel: +91 2225767207

${ }^{8}$ Wadhwani Research Center for Bioengineering, Indian Institute of Technology Bombay, Mumbai, India

$\dagger$ Electronic supplementary information (ESI) available. See DOI: $10.1039 / \mathrm{c} 7 \mathrm{ra05039a}$ nanofibers to heal bone defects faster. To mimic natural extracellular matrix (ECM) architecture of bone, an open porous three-dimensional interconnected fibrous network is required with the fiber diameter ranging from 50 to $1000 \mathrm{~nm}$ with porosity greater than $90 \%$ are considered ideal properties for bone grafts for non-load bearing applications. Electrospun nanofibers, by virtue of their high surface area and porosity, provide enhanced cell adhesion, proliferation and differentiation. Nanofiber structural similarities to 3D architecture of natural ECM provide a favorable environment ranging from micro-nano for cells to proliferate and perform their regular functions. ${ }^{1}$ A variety of biomaterials have been considered for this application ranging from natural to synthetic polymers reinforced with metal to ceramics. The lack of control of degradation over ceramics in biological milieu and difficulties in processing them into desired shapes have been problem for their widespread use in bone tissue engineering. For these reasons, polymeric materials have received attention and are currently widely studied for this application. PCL (polycaprolactone), PGA (poly(glycolic acid)), PLA (poly(lactic acid)), and their copolymer PLGA are the most popular synthetic 
polymeric materials used and are approved by Food and Drug Administration (FDA) for use in humans. ${ }^{2}$ PCL being the slowest degrading polymer having the lowest inflammation and moderate $\mathrm{pH}$ increase during degradation has been the preferred choice for bone regeneration.

Ceramics have been widely used for bone substitution/ regeneration in clinical practice. Naturally occurring hydroxyapatite (HA) (e.g., coralline HA) or its synthetic composites such as chemically formed HA or $\beta$-tricalcium phosphate ( $\beta$-TCP) have been widely used for bone tissue engineering applications due to their osteoconductive and osteopromotive properties. Several studies have reported that by using ceramics with or without bone marrow cells or stem cells have yielded higher bone regeneration; however major drawbacks including brittle nature of ceramics and their low mechanical stability prevent their use in the regeneration of large bone defects. The in vivo influencing factors, such as osteoclastic activity and degradation/dissolution rates, have been difficult to extrapolate from in vitro data. This represents a problem because if it degrades too fast, mechanical stability of the construct at the site is compromised, which is low by itself. If there is higher leaching or dissolutions from bone graft of calcium and phosphorus, it may lead to a dramatic increase in concentrations of extracellular milieu which can cause cellular death. ${ }^{3}$ Alone, synthetic ceramics neither promote osteogenic or osteoinductive properties nor provide immediate mechanical support. When inserted at a defect surrounded by healthy bone, osteoid formation is reported on the surfaces of the ceramic in the absence of a soft tissue interface. ${ }^{4}$ Consequently, the osteoid mineralizes to form immature bone, and thus, the resulting new bone undergoes remodeling to form a mature bone.

Studies in the past have demonstrated that sintered calcium (Ca) phosphorus (P)-based bioceramics have slow degradability and might be present at the site for more than one year. ${ }^{5}$ Degradation plays an important role in bone regeneration since faster degradation rate can cause the following problems in clinical use: implant failure due to poor bone implant contact surface and slow degradation rate leading to slow bone formation rate. In an in vitro study by our group, we compared the degradation of PCL-HA and PCL-HS to show that increasing the percentage of HS in the nanofiber leads to increase in degradation rate. ${ }^{6}$ To achieve optimal bone strength and faster bone regeneration, several trace impurities have been introduced in hydroxyapatite to suit various application ranging from regeneration to osteointegration. Hench et al. developed an advanced biomaterial, $\mathrm{SiO}_{2}-\mathrm{CaO}-\mathrm{Na}_{2} \mathrm{O}-\mathrm{P}_{2} \mathrm{O}_{5}$ (silicon ( $\mathrm{Si}$ ), oxygen $(\mathrm{O})$, calcium (Ca), phosphorus (P)) glass in the 1970s and showed that Ca-Si-based bioactive glass is capable of inducing HAp crystal layer on the material. ${ }^{7}$ Most studies have shown that modified HA or its substitutes with trace impurities provide good biocompatibility and bioactivity. Similar supportive studies have been conducted effect $\beta$-TCP has been compared with that of calcium silicate on rabbit calvarial defects over 16 weeks, and the results show that significantly higher bone formation was observed in calcium silicate and it also resorbed faster than $\beta$-TCP. This shows that silica plays a major and distinct role in accelerating bone formation. ${ }^{8}$
Zinc $(\mathrm{Zn})$ is an essential trace element and gives stimulatory effect for bone formation and mineralization; it has also shown to have bone anti-resorptive property. ${ }^{9}$ Zinc studied alone or in combination with magnesium or strontium-incorporated HA in $10 \%$ amount in implants in osteopenic rats show that significantly higher bone formation was achieved with zinc in comparison with HA alone though the highest bone formation was shown after strontium incorporation. ${ }^{\mathbf{1 0}}$ Earlier studies of $\mathrm{Zn}$ doped $\mathrm{CaSiO}_{3}$ showed that incorporation of $\mathrm{Zn}$ in material promotes human bone osteoblastic-like cell proliferation and when quantity of $\mathrm{Zn}$ exceeds $20 \%$, there is no effect on proliferation but ALP activity increases up to Zn quantity of $50 \% .^{11} \mathrm{~A}$ study conducted by Ramaswamy et al. have compared $\mathrm{CaSiO}_{3}$ with hardystonite $\left(\mathrm{Ca}_{2} \mathrm{ZnSi}_{2}-\mathrm{O}_{7}\right)$ with respect to biological response, and they have shown that addition of $\mathrm{Zn}$ to $\mathrm{Ca}-\mathrm{Si}$ group enhances human osteoblast-like cell proliferation and differentiation. ${ }^{\mathbf{1 2}}$

Our objective is to evaluate bone regeneration potential and safety of nano-agglomerated HA and HS reinforced in PCL nanofibers. We study bone response and rank PCL-HA and PCL-HS in terms of micro-CT (computed tomography) analysis, histology, hematology and serum biochemistry parameters.

\section{Materials and methods}

In this section, method of scaffold fabrication is given in brief. Process of animal surgery and scaffold implantation in rat calvaria is described in detail. Post-sacrifice evaluation methods, namely blood and serum analysis, and micro-CT are also elaborated in detail.

\subsection{Materials}

Polycaprolactone (PCL) (Sigma Aldrich, St. Louis, MO, USA) with an average molecular eight of $80000 \mathrm{Da}, 1,1,1,3,3,3$-hexafluoro2-propanol (HFIP); and hydroxyapatite powders were provided by Plasma Biotal Ltd., UK, tetraethyl orthosilicate $\left(\left(\mathrm{C}_{2} \mathrm{H}_{5} \mathrm{O}\right)_{4} \mathrm{Si}\right.$, TEOS), zinc nitrate hexahydrate $\left(\mathrm{Zn}\left(\mathrm{NO}_{3}\right)_{2} \cdot 6 \mathrm{H}_{2} \mathrm{O}\right)$, calcium nitrate tetrahydrate $\left(\mathrm{Ca}\left(\mathrm{NO}_{3}\right)_{2} \cdot 4 \mathrm{H}_{2} \mathrm{O}\right)$ and nitric acid $\left(\mathrm{HNO}_{3}\right)$ were purchased from Merck, India. Water was distilled and deionized (DDW) using a Milli-Q system (Millipore, MA, USA).

\subsection{Synthesis of hardystonite (HS) powders}

Hardystonite powders were prepared as described by the sol-gel process. ${ }^{26}$ Mixture of TEOS, DDW and $2 \mathrm{M} \mathrm{HNO}_{3}$ were stirred at $300 \mathrm{rpm}$ for $30 \mathrm{~min}$ in a molar ratio of $\mathrm{TEOS} / \mathrm{H}_{2} \mathrm{O} / \mathrm{HNO}_{3}=$ $1: 8: 0.16$. After completion of the above process, $\mathrm{Zn}\left(\mathrm{NO}_{3}\right)_{2}$ $\cdot 6 \mathrm{H}_{2} \mathrm{O}$ and $\mathrm{Ca}\left(\mathrm{NO}_{3}\right)_{2} \cdot 4 \mathrm{H}_{2} \mathrm{O}$ were added into the mixture in a molar ratio of TEOS $/ \mathrm{Zn}\left(\mathrm{NO}_{3}\right)_{2} \cdot 6 \mathrm{H}_{2} \mathrm{O} / \mathrm{Ca}\left(\mathrm{NO}_{3}\right)_{2} \cdot 4 \mathrm{H}_{2} \mathrm{O}=2: 1: 2$ and all reactants were stirred again for $5 \mathrm{~h}$ at room temperature. The solution was kept for ageing at $60{ }^{\circ} \mathrm{C}$ for $24 \mathrm{~h}$ and further dried at $120^{\circ} \mathrm{C}$. After drying, the powder was finally calcined at $1250{ }^{\circ} \mathrm{C}$ in a glass furnace for $2 \mathrm{~h}$, followed by ball milling.

\subsection{Processing of nanofibrous scaffolds by electrospinning}

PCL was dissolved in 1,1,1,3,3,3-hexafluoro-2-propanol (HFIP) to prepare (w/v) solution for electrospinning. To fabricate PCL-HA 
scaffolds, PCL was dissolved in HFIP to obtain a $15 \%(w / v)$ final solution, and for composite filler HA, HS was added to $40 \%$ wt of PCL $(\mathrm{w} / \mathrm{w})$. The polymer solution was stirred overnight at room temperature before electrospinning and filled in a $5 \mathrm{ml}$ plastic syringe with blunt-ended metallic needle (21 Gauge) (BD, India); care was taken to avoid air bubbles. The syringe filled with the polymer solution was loaded into a syringe pump (New Era Pump System Inc., USA) and the syringe needle tip was connected to the positive output of a high voltage power supply (Gamma High Voltage, USA), while an aluminium foil covered collector was grounded. The needle tip to collector distance was kept at $12.5 \mathrm{~cm}$, the voltage at $14 \mathrm{kV}$ and the flow rate was $0.7 \mathrm{ml}$ $\mathrm{h}^{-1}$. The entire process was conducted in a fume hood at room temperature between $22-27^{\circ} \mathrm{C}$ and $55 \%$ relative humidity. The electrospun scaffold was kept under vacuum overnight before carrying out any further experiments.

\subsection{Morphological analysis by scanning electron microscopy (SEM) and transmission electron microscopy (TEM)}

Morphological analysis of electrospun scaffolds and nanoparticles was performed using a scanning electron microscope (FEI, Quanta 200 (D 7548)) at an accelerating voltage of $15 \mathrm{kV}$ in SEM. All spinning parameters were tested in triplicate and 50 fibres per SEM micrograph were measured. Transmission electron microscopy (TEM, JEM 2100 ultra HRTEM, Jeol, Japan) was employed to study dispersion of filler particles in polymer fibres at $200 \mathrm{kV}$ voltage.

\subsection{Degradation profile of calcium, phosphorus and zinc by inductively coupled plasma atomic emission spectroscopy (ICP-AES)}

Extract of the nanofibrous scaffold was prepared for detection of $\mathrm{Ca}, \mathrm{P}$ and $\mathrm{Zn}$ for leaching. Preparation was done following the standard method described in ISO-10993-12 where polar extractant in a mixture of $20: 80 \mathrm{EtOH} /$ water was used for leaching; this was followed by incubation at $50{ }^{\circ} \mathrm{C}$ for $72 \mathrm{~h}$ at $100 \mathrm{rpm}$. A weighed quantity of the scaffold was immersed in the extractant in the mass to extractant ratio of $3 \mathrm{mg} \mathrm{ml}^{-1}$ and was incubated under above mentioned conditions for four timepoints, 12, 24, 48 and $72 \mathrm{~h}$. The extracts were then collected by simple decantation and measured properly for ICP-AES sample preparation, where a measured volume of extract with $50 \%$ concentrated nitric acid was added in a beaker and heated at $102{ }^{\circ} \mathrm{C}$ for $3 \mathrm{~h}$, followed by 10 -fold dilution.

\subsection{Animal study}

2.6.1 Animals and housing. All animal experiments were performed with approval from Institutional Animal Ethics Committee of the National Institute for Research in Reproductive Health (NIRRH Approval no. NIRRH/IAEC/04/10). The study performed in compliance with the guidelines of the Committee for the Purpose of Control and Supervision of Experimental Animals (CPCSEA), India. Six- to eight-week-old healthy adult Wistar male rats with an average body weight (BW) of $350 \pm 30 \mathrm{~g}$ were used in this study. All animals used in the study were bred in the institute premises of NIRRH and were housed in polypropylene cages with a maximum of one animal per cage. The temperature of the cages was controlled temperature of $23 \pm 1{ }^{\circ} \mathrm{C}$, humidity of $55 \pm 5 \%$ and a $14 \mathrm{~h}$ light/10 h dark cycle. The cages contained autoclaved corn cob as bedding that was replaced every week. Throughout the study, the animals were provided with soy-free, in-house prepared pelleted feed and filtered drinking water ad libitum.

2.6.2 Test animals and husbandry. For efficacy and safety evaluation, 6 month-old Wistar male rats were used in the study. The animal identification was performed as per the method described in the institute guidelines. Animals were transferred from the breeding room to experimental room and kept for acclimatization at least five days prior to the start of the study. The temperatures in the experimental animal room were maintained between $20^{\circ}$ and $22{ }^{\circ} \mathrm{C}\left( \pm 3{ }^{\circ} \mathrm{C}\right)$. The relative humidity was maintained between $50 \%$ and $60 \%$. Artificial lighting was provided that included 10 hours light and 14 hours dark. All animals were given only pelleted feed prepared at the institute and drinking water was supplied ad libitum.

2.6.3 Study design and surgical procedures. We tested three kinds of scaffolds, namely polycaprolactone (PCL), polycaprolactone-hydroxyapatite (PCL-HA) and polycaprolactonehardystonite (PCL-HS). The scaffolds were implanted on the defect created along the sagittal line for the period of 6 and 12 weeks.

A sterile surgical technique was used for the experiment. Before implantation, all scaffolds were sterilized by $70 \%$ ethanol and ultraviolet light in a laminar hood. Animals were anesthetized by intraperitoneal injection with a mixture of ketamine HCl (60 mg kg ${ }^{-1}$, Intas Pharma Ltd., Ahmedabad, Gujarat, India) and xylazine ( $7 \mathrm{mg} \mathrm{kg}^{-1}$, Themis Medicare Ltd., Vapi, Gujarat, India). Once sedated, the incision site was shaved and sterilized with betadine (G.S. Pharmbutor Pvt. Ltd., Rajasthan, India) and $70 \%$ ethanol. The scaffolds were implanted on the defect created along the sagittal line for the period of 6 and 12 weeks. The soft tissue and periosteum were elevated to expose the calvarial surface on the sagittal suture line. Full-thickness defects $(8 \mathrm{~mm}$ diameter) were created with a trephine bur attached to a dental hand piece with a micromotor in the parietal bones, with the sagittal suture line in the middle (ESI Fig. $1 \dagger) .{ }^{13}$

Constant sterile saline was irrigated to avoid heat generation during surgery. Extreme care was taken to avoid injury to both the sagittal suture and the brain. After removal of the trephined calvarial bone, PCL, PCL-HA and PCL-HS scaffold was implanted into the defect in respective animal groups. No scaffold was implanted for animals in the SHAM group. The pericranium and skin were closed in layers. Each rat was individually caged after surgery and observed for clinical signs and symptoms (Table 1).

\subsection{Quantitative performance analysis}

2.7.1 Micro-CT analysis. Rat head bone after euthanasia was fixed with formaldehyde and washed before scanning on an animal micro-computed tomography (CT) scanner (GE FLEX Triumph micro-PET/SPECT/CT scanner) using an X-ray tube potential of $75 \mathrm{kV}$ and a tube current of $100 \mu \mathrm{A}$. The scanner 
Table 1 Experimental groups: SHAM (empty defect), polycaprolactone $(P C L)$, polycaprolactone-hydroxyapatite $(P C L-H A)$ and polycaprolactone-hardystonite (PCL-HS) at 6 and 12 weeks; $N$ factor is 3

\begin{tabular}{llll}
\hline & & \multicolumn{2}{l}{ Timepoints } \\
\cline { 3 - 4 } Sr. no. & Group name & $T=6$ weeks & $T=3$ weeks \\
\hline 1 & SHAM (control) & $N=3$ & $N=3$ \\
2 & PCL & $N=3$ & $N=3$ \\
3 & PCL-HA & $N=3$ & $N=3$ \\
4 & PCL-HS & $N=3$ & $N=3$
\end{tabular}

used an X-ray source and a detector system that rotated around a bed holding the calvaria. Data were acquired over $360^{\circ}$, producing 1024 views. CT images were reconstructed using a modified cone-beam algorithm with an isotropic voxel spacing of $0.045 \times 0.045 \times 0.045 \mathrm{~mm}^{3}$ over a region of interest. In addition, micro-CT scans were also performed on rat heads harvested from the SHAM control animals as the negative control. Micro-CT scanning protocol was optimized by varying voltage $(70,75$ and $90 \mathrm{kV})$ to acquire a high-quality image, which we observed at $75 \mathrm{kV}$ for 10.50 time with 1024 slices. Parameters and conditions were kept constant for all samples.

2.7.2 Histological analysis. For histological analysis, calvaria were decalcified with $\mathrm{HCl}$ and EDTA (ethylenediaminetetraacetic acid) for 3 weeks and processed and embedded in paraffin. Each block was trimmed from the corners and sectioned with a microtome (Leica RM2156; Leica, Wetzlar, Germany) equipped with a tungsten carbide knife of 10 micron thickness. The sections were stained with hematoxylin and eosin (H\&E). The morphological evaluation of the tissue response in the different groups was assessed using an optical microscope (Carl Zeiss, Berlin, Germany).

\subsection{Blood collection, hematology and serum biochemistry}

A complete blood count (CBC) was performed for clinical pathology and diagnosis of any inflammatory responses after implantation of scaffolds for 6 and 12 weeks. After the end of the respective time point ( 6 and 12 weeks), animals were bled from the retro-orbital sinus, and $1 \mathrm{ml}$ blood was collected in EDTA coated vials for hematology analysis and $2 \mathrm{ml}$ blood was collected in plain vials to obtain serum for biochemical estimations. Heparin ( $2 \mathrm{IU} \mathrm{ml}^{-1}$ ) containing vials were used for hematological parameters like hemoglobin $\left(\mathrm{Hb} ; \mathrm{g} \mathrm{dl}^{-1}\right)$, packed cell volume (PCV; \%), total red blood cells (RBCs), total white blood cells (WBCs), absolute erythrocyte indices, and differential WBC count. Differential WBC count (lymphocytes, monocytes, neutrophils, eosinophiles and basophils) was performed using blood smears. CBC was performed using an Abacus (Diatron MI PLC, Hungary) hematology analyzer, while serum biochemistry evaluation was conducted on an Erba Chem 7 (Erba Mannheim, Germany) semi-auto analyzer with commercial reagent kits where in second vial without heparin was used for serum biochemistry parameters such as serum glutamic pyruvic transaminase (SGPT), serum glutamic oxaloacetic transaminase (SGOT), alkaline phosphatase (ALP), creatine and urea.

\section{Results and discussion}

\subsection{Morphological analysis}

A biomaterial woven into an artificial scaffold for tissue engineering should be biocompatible and possess an ability to regenerate bone. We studied the efficacy and safety of PCL, PCL-HA and PCL-HS in critical-sized defects in rats. The rat calvarial model with critical defects of $8 \mathrm{~mm}$ was chosen for the study because of the easy approval, availability and delayed nonunion healing in rats at the defect site. Calvaria model with a critical size was chosen because the calvaria has poor blood supply, relative deficiency of bone marrow and physiological similarity with membranous maxillofacial bone formation. A critical-sized defect is defined as a defect that is 1.5-3.0 times smaller than the diameter of the bone and should not heal more than $30 \%$ in a 24 week period. ${ }^{\mathbf{1 4 , 1 5}}$

The scaffolds fabricated using electrospinning, as shown in Fig. 1, were smooth with average pore size in the range of $3.07 \pm$ $1.08 \mu \mathrm{m}$ (PCL), $2.96 \pm 1.07 \mu \mathrm{m}$ (PCL-HA) and $3.14 \pm 1.33 \mu \mathrm{m}$ (PCL-HS), so there was no significant difference between surface pore size of any group among the nanofiber scaffolds. TEM images of the scaffolds show the presence of HA and HS in the fibers in nano-particulate form and the presence of HA and HS was quantified by thermogravimetric analysis and was shown to be $30 \%$ in an earlier paper. ${ }^{6}$ The longest length of particle for HA and HS was calculated to be $2.52 \pm 0.74 \mu \mathrm{m}$ and $375 \pm 100 \mu \mathrm{m}$, respectively, from SEM micrographs for the aspurchased and synthesized powder. The EDS analysis confirmed peaks corresponding to major elements such as oxygen, calcium and phosphorus in HA along with silicon and zinc in HS (ESI Fig. 2†). HA and HS were confirmed to have a crystal structure by TEM micrographs. The diffraction analysis of HA gave perfect single-crystalline SAED patterns, which reveal a material with highly ordered micron agglomerate crystal structure, whereas HS gave spotty-ring SAED patterns with multiple planes. HA and HS showed polycrystalline pattern with the most important interplanar spacing (Fig. 2). ${ }^{\mathbf{1 6}}$

In vitro study showed that HS-containing composite of PCL as nanofibrous scaffolds exhibited superior physicochemical and biological performance in comparison to HA-containing composite PCL scaffolds. Our finding from an earlier published study also demonstrated superior mechanical strength of PCL-HS scaffolds as compared to PCL-HA scaffolds. Proliferation and cellular infiltration of mE-ASCs among all scaffolds were also found to be better in PCL-HS scaffolds. ALP activity and mineralization were also found to be significantly higher in PCL-HS scaffolds as compared to PCL-HA scaffolds, which did not translate into the present in vivo study. ${ }^{6}$

\subsection{Degradation profile of calcium, phosphorus and zinc by inductively coupled plasma atomic emission spectroscopy (ICP-AES)}

Inductively coupled plasma atomic emission spectroscopy was used to assess the release of calcium, phosphorus and zinc from the nanofibrous scaffold in comparison to those containing HA and HS over the 4 timepoints namely 12, 24, 36 and $72 \mathrm{~h}$. 

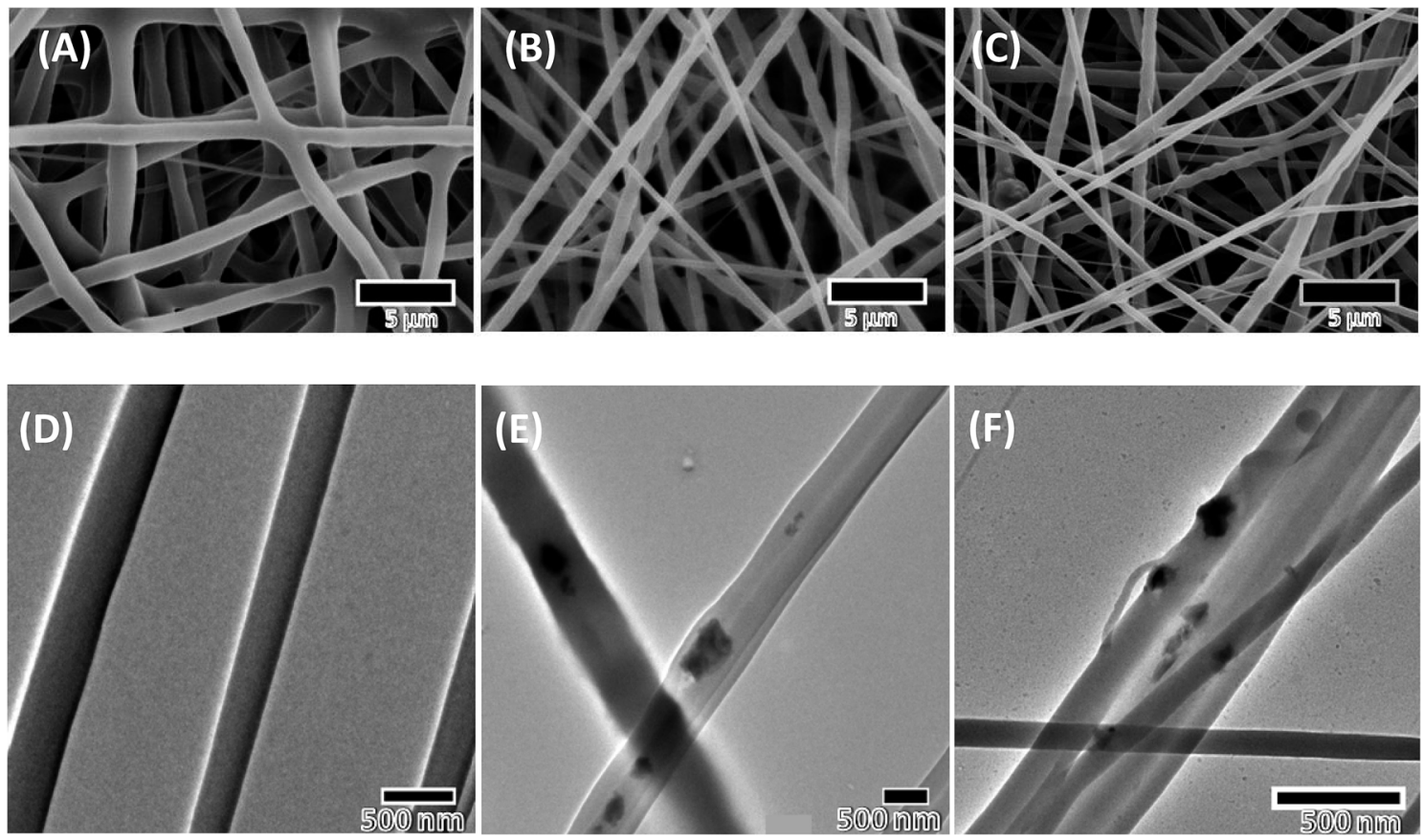

Fig. 1 SEM and TEM images of 3 nanofibrous scaffolds studied: (A) polycaprolactone (PCL), (B) polycaprolactone with hydroxyapatite (PCL-HA), and $(C)$ polycaprolactone with hardystonite (PCL-HS). (D) TEM images of PCL, (E) TEM images of PCL-HA and (F) PCL-HS. All Scaffolds were fabricated using electrospinning with similar parameters, giving thinner fibers for $\mathrm{PCL}-\mathrm{HS}$ and $\mathrm{PCL}-\mathrm{HA}$.
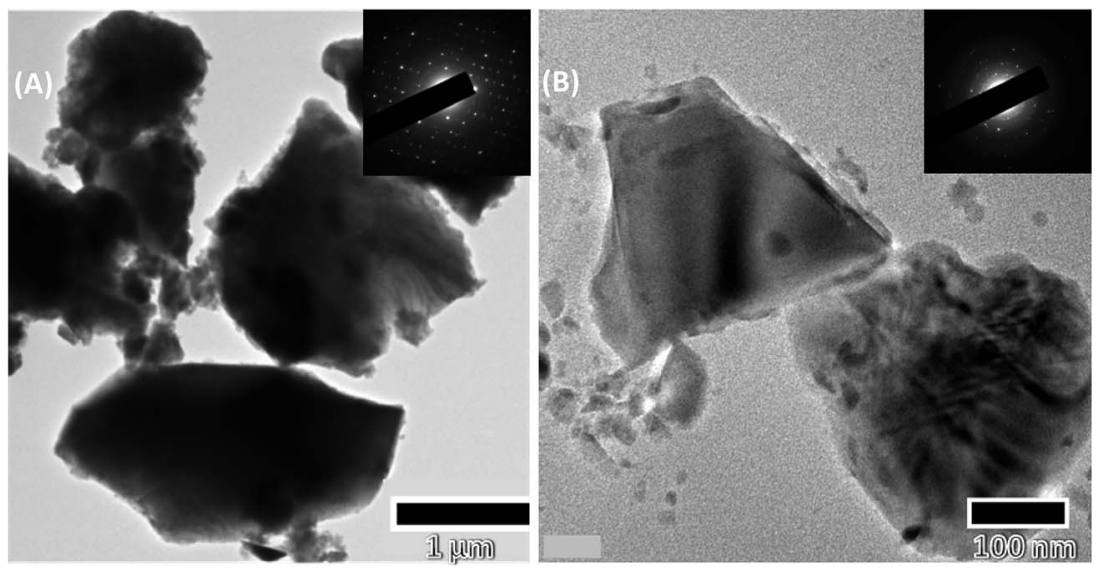

Fig. 2 TEM image with diffraction patterns of nanoparticles after sonication for 30 min used for electrospinning (A) hydroxyapatite (HA) and (B) hardystonite (HS).

Ethanol to water $(80: 20 \%)$ was used to leach the scaffolds since a polymeric system leaches more as compared to $20 \mathrm{mM}$ sodium acetate. ${ }^{16}$ Phosphorous and zinc release, which is characteristic of HA and HS, was in higher ppm concentration in comparison to that for filler-reinforced fibers. Phosphorus release from PCL-HS and HS was found to negligible since they did not contain these elements. There was a significant difference between the release profile of calcium from HA and from HS, since HS is more brittle, which may be the reason for high release of calcium from HS, though there was no difference in cumulative quantitative release of calcium from PCL-HA and PCL-HS (Fig. 3). Zinc release quantification revealed that there was negligible release from PCL-HA and HA, but at $72 \mathrm{~h}$, the zinc release was similar from both PCL-HS and HS.

\subsection{Performance analysis}

The regenerated bone from scaffolds fabricated with PCL and their composites was scanned at the defect site after each time points using micro-CT (optimized protocol; refer to Material and methods, Micro CT). MicroView (Parallax Innovations, ON, Canada) was used for analysis of bone growth and density. On comparing the performance of PCL, PCL-HA, and PCL-HS nanofibrous scaffolds with SHAM for rat calvaria with $8 \mathrm{~mm}$ 

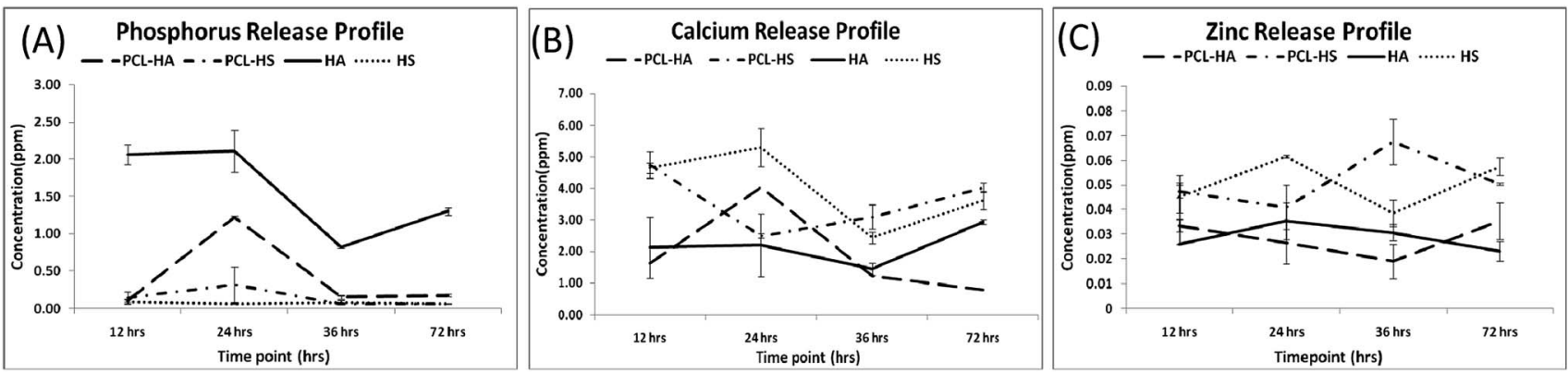

Fig. 3 (A) Phosphorous, (B) calcium and (C) zinc release profiles from polycaprolactone-hydroxyapatite (PCL-HA), polycaprolactone-hardystonite $(\mathrm{PCL}-\mathrm{HS})$, hydroxyapatite $(\mathrm{HA})$ and hardystonite $(\mathrm{HS})$ at $12,24,36$ and $72 \mathrm{~h}$.

critical-sized defect through reconstructed images, we found that bone formation (BV/TV \%) in all scaffolds was higher than in SHAM at both the 6 and 12 week timepoints (Fig. 4). There was a significant difference observed between the 6 and 12 week timepoint in all groups. Bone growth in PCL-HA and PCL-HS groups was higher than that in SHAM and PCL groups at the 12 week timepoint, which points to the advantage of adding nanofillers to nanofibers for higher bone regeneration. A similar study of HA and $\beta$-TCP incorporated as nanofillers in PCL nanofibers have shown to positively affect the differentiation property via upregulation of runt-related transcription factor 2 (Runx-2), bone sialoprotein (BSP) and type I collagen (Col-1) genes through the likely coupled effect of an ECM-like nanostructure and composite of scaffolds during differentiation of human stem cells towards bone lineage. ${ }^{17}$ There was significant difference observed in BVF\% for all groups SHAM, PCL, PCL-HA and PCL-HS as compared to adjacent original bone at 6 and 12 weeks. Bone growth showed no difference in terms of amount of bone regenerated in PCL and SHAM groups at 12 weeks, whereas there was significant difference between SHAM and PCL groups and PCL-HS group, which shows the capability of HS-reinforced substitutes. However, bone volume formation (BV/TV\%) in the SHAM and PCL groups was comparable with that in the PCL-HA group, which is in sync with the results of Zhang et al., who reported that new regenerated bone area was not significant when HA and HS coating on metal implants were compared; nevertheless, bone implant contact (BIC) for Sr-HS and HS groups were significantly higher

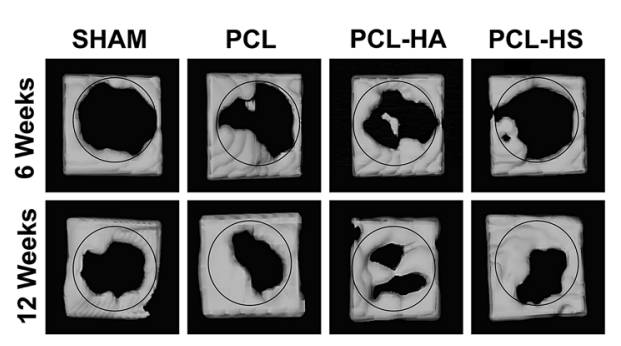

Fig. 4 Reconstructed 3D micro-CT images of rat calvaria implanted with PCL, PCL-HA and PCL-HS scaffolds after 6 weeks and 12 weeks along with those with SHAM. BV/TV ratio of PCL-HS showed significant difference compared to that of SHAM and PCL at 12 weeks. Both $\mathrm{PCL}-\mathrm{HA}$ and $\mathrm{PCL}-\mathrm{HS}$ were equally effective. than those seen in the HAp group. ${ }^{18}$ The difference between two studies can be ascribed to entrapment of HA within the nanofibers as compared to the native HA. ${ }^{19}$ The shape and size of HA in nanoforms and other ceramics have been shown to affect bone formation..$^{20,21}$

Differences in BV/TV\% can also be attributed to difference in fiber diameters of PCL, PCL-HA, and PCL-HS due to viscosity of the solution, keeping other parameters same. ${ }^{22}$ The diameter of PCL fiber in (mean + SE) was $1.17+0.03 \mu \mathrm{m}$, whereas those of PCL-HA and PCL-HS were $0.75+0.04 \mu \mathrm{m}$ and $0.50+0.03 \mu \mathrm{m}$ (Fig. 1), respectively, with significance $(p<0.05)$ among all groups. Other studies observed complex dependence of cell proliferation on fiber diameter between 428 and $1647 \mathrm{~nm}$ diameter scaffolds of PCL at over a 7 day time period and found that a $428 \mathrm{~nm}$-diameter scaffold showed higher proliferation. ${ }^{23}$ The role of chondrogenesis was also explored using electrospun fibrous scaffolds with fiber diameters ranging from $300 \mathrm{~nm}$ to $1 \mu \mathrm{m}$ on which bovine articular chondrocytes were studied for 7 and 14 days. An upregulation in gene expression of collagen II/collagen I ratio was 2 times higher with smaller diameter nanofibrous sheets, which correlated with our result. ${ }^{24}$ This upregulation may also be responsible for the difference among the groups.

Bone quality analysis in terms of bone mineral density, tissue mineral content and tissue mineral density was also performed using MicroView as a function of $\mathrm{HU}$ number to compare the quality of bone formed at 6 and 12 weeks among different groups (ESI Table $1 \dagger$ ). The combined density of a region of interest that contains both bone and soft tissue is measured as "bone mineral density" or BMD. BMD is related to the amount of bone within a bone-soft tissue region, but does not give information about the material density of the bone itself. The density measurement calculates only the volume within the calcified bone tissue, such as cortical bone, excluding surrounding the soft tissue, is called "tissue mineral density" or TMD. ${ }^{25}$ There was no difference between bone mineral content, bone mineral density, tissue mineral content and tissue mineral density among groups at all timepoints, which shows that bone deposited had equal mineralization in all groups. Whereas there was significant difference in BMD between all treated sites to the adjacent original bone, there was no difference observed in TMD at 6 and 12 week timepoint. TMD is characteristic of 
cortical bone which forms first, specially at blood contact surfaces. Therefore TMD doesn't show any significant difference. A similar trend was observed when peripheral blood mesenchymal stem cells/self-assembly peptide/PLGA nanofibers were implanted in critical-sized rat calvarial model with SAP/PLGA as the control; there was no significant difference observed in BMD but there was a significant difference in bone formation at the defect site. ${ }^{26}$ PCL-HA and PCL-HS formed bone similar TMD as comparable to the adjacent original bone (Fig. 5). Results from density analysis showed that there was no significant difference between bone formed without the nanofiber scaffolds and when it was facilitated by the PCL based composite nanofibrous scaffold.

Quantification of micro-CT generated images for relative counts of spongial and cortical bone was performed by converting the range of X-ray intensity originally measured in Hounsfield unit (HU) into the tissue types (Fig. 6); this is achieved by voxel-level segmentation of HU units into discretized levels. To quantify the count, two thresholds are identified: (1) spongial bone limit between soft and cortical bone and (2) cortical bone limit. MIMICS ${ }^{\circledR}$ was used to obtain histograms because of its inbuilt threshold software, which in our case was determined to be 225 to 661 for spongial and 662 to 1988 for cortical. ${ }^{27}$ The histogram obtained with the frequency gives the amount and peak intensity for qualitatively understanding the bone. The outcome shown in Fig. 6 is a time series of the spatial distribution, the relative amount of two tissue types, and a time series of HU units to voxel counts, which is useful in understanding bone healing and compaction. With respect to cortical bone, PCL-HA and PCL-HS groups had more counts than PCL and SHAM groups. PCL-HS group was found to have the least

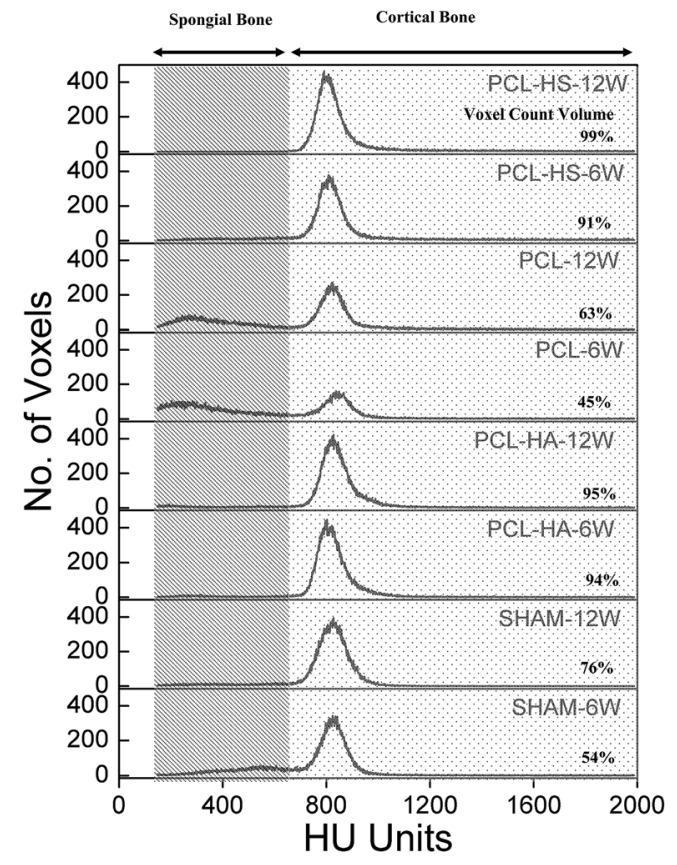

Fig. 6 MIMICS analysis: spongial bone (225-661 Hounsfield units (HU)) and cortical bone (662-1988 HU) represented by dotted and line patterns. $\mathrm{PCL}-\mathrm{HA}$ and $\mathrm{PCL}-\mathrm{HS}$ groups had larger voxel count percentage in cortical bone region that PCL and SHAM groups at 12 weeks.

spongial bone count and most cortical bone count, which shows the higher quality of bone compared to other groups.

Biocompatibility is considered to be one of the important criterions for long-term host-tissue implanted material
(A)

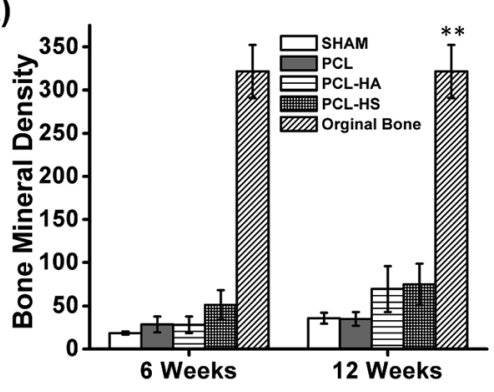

(C)

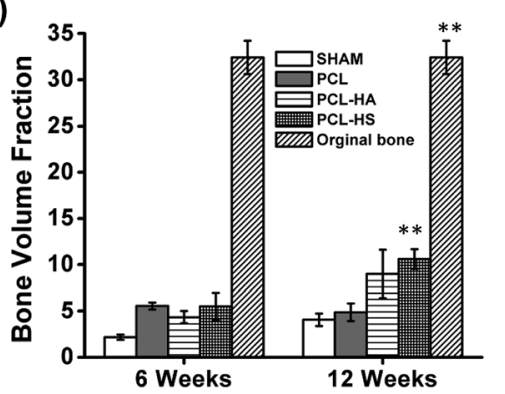

(B)

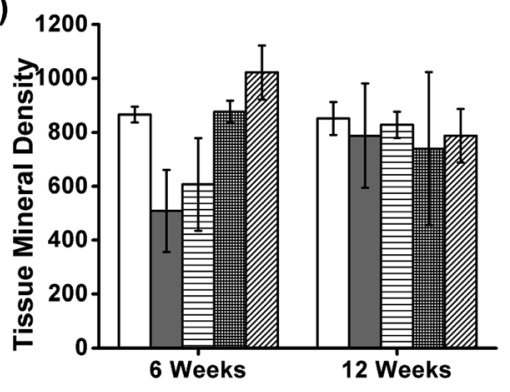

(D)

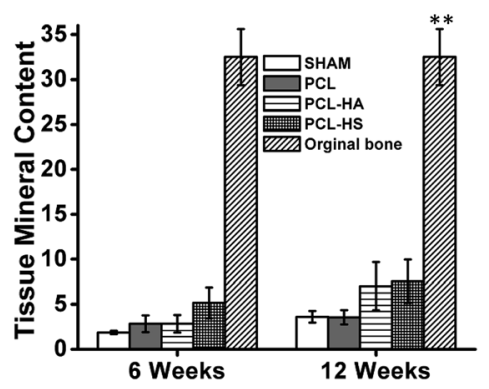

Fig. 5 Quantitative comparison from micro-CT analysis: (A) bone mineral density; (B) tissue mineral content; (C) bone volume/total volume fraction; and (D) tissue mineral density among different groups with comparison to bone adjacent to the defect (original bones) at 6 and 12 weeks. Significant difference $(p<0.05)$ in bone volume fraction is observed between PCL-HS and PCL and SHAM at 12 weeks. No significant difference in bone mineral density, tissue mineral content and tissue mineral density is observed among groups at 12 weeks. 
interaction. This inflammation interaction and its role in bone fracture healing was elaborately understood by Hench in 1994 . Variation in blood parameters is being studied over 6 and 12 weeks to observe partial resorption of material in the animal model. $^{28}$

Histology results were consistent with micro-CT results. The histology images showed high cell activity in all scaffolds at the interface of the defect. They also showed the formation of more trabecular bone as neo-bone at the defect interface in PCL-HS group as compared to that in all other groups at 6 weeks (Fig. 7), which is the bone forming block. ${ }^{29}$ At the 12 week time point, we observed high inflammation activity with fibrous tissue in the SHAM group, porous bone being formed at the defect interface in the PCL group and broader bones being formed in the PCLHA and PCL-HS group, which show the ability of integration of nanofiber with the surrounding bone (Fig. 7). The occurrence of direct contact at the interface between bone formed by scaffolds and surrounding bone tissue was used as another indicator of bone biocompatibility. ${ }^{30}$ Tissue-biomaterial interfacial bonding has shown to play an important role in the success of bone grafts used for load-bearing in orthopedic and dental surgery. The interfacial bonding in the PCL-HS group to the surrounding bone was significantly higher than that in PCL-HA group, reflecting the ability of $\mathrm{HA}$ and $\mathrm{HS}$ to induce osseointegration without any infiltration of fibrous tissue between the old and new bone. At the 12 week timepoint, small scattered bone blocks were observed near the periphery site with inflammatory cells in the SHAM group, whereas in the PCL group, neo-bone was observed at 6 weeks surrounded by inflammatory cells, which became thicker bones osteointegrated with old bones touching at few periphery points at the defect site at 12 weeks. The presence of these inflammatory cells in the SHAM and PCL groups is indicative of a reaction between the host reaction and biomaterials at 6 weeks. ${ }^{31}$ In the PCL-HA group, lamellar neo-bone was observed with the material present at the defect site, as shown in Fig. 8 at 6 weeks. At 12 weeks, PCL-HA group subjects showed complete integration of bone defect site with a thick band of bone, whereas in the PCL-HS group, bone formation was observed in 2 bands, where the first band at the site integrated with the old bone and the second band of neo-bone was laid down; this group also showed complete integration at the 6 week timepoint.

\subsection{Biocompatibility in vivo}

3.4.1 Gross observation. Wound healing in this study is a four-stage process: the first stage corresponds to the inflammatory process where biocompatibility plays the first and larger role; the second to the proliferative stage; the third to the differentiation stage of stem cells and premature bone formation and the fourth to the remodeling of premature bone to mature bone. ${ }^{32}$ None of the groups on implantation showed any mortality or clinical signs of disorder or discomfort. No behavioral changes or visible signs of physical impairment or neurological toxicity were noticed during the 12 week observation period. Macroscopic analysis of the implant sites demonstrated comparable scar formation and subsequent healing processes in all four groups. The macroscopic appearance of the scar created by excision of $3 \mathrm{~cm}$ of dermis and epidermis on the
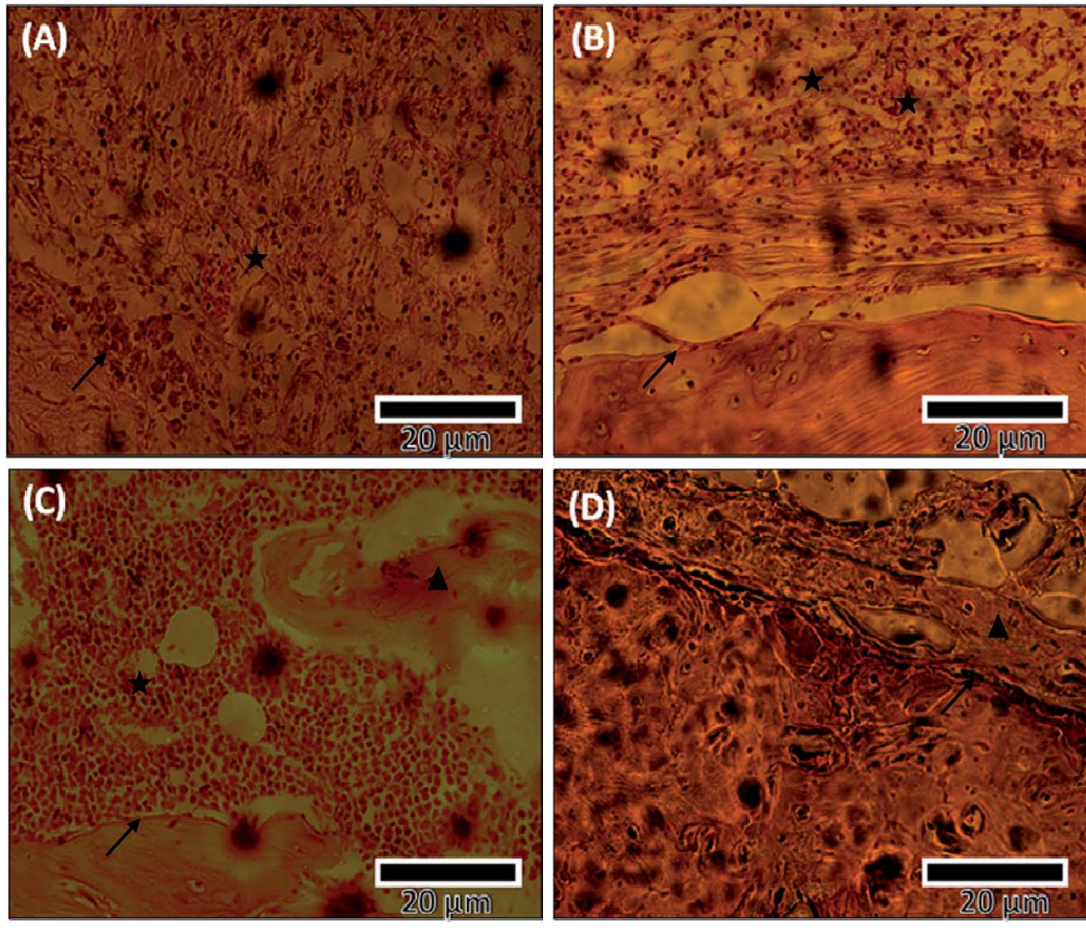

Fig. 7 Histology of treated site and control after 6 weeks using hematoxylin and eosin (H\&E) staining: (A) SHAM; (B) PCL; (C) PCL-HA and (D) $\mathrm{PCL}-\mathrm{HS}$ higher osteointegration and bone formation were observed in the PCL-HS group at the defect site as compared to those in other groups. Higher inflammation was observed at the defect site in the SHAM group. $\triangle$ Defect site boundary, $\star$ inflammatory cells, $\boldsymbol{\Delta}$ neo-bone. 

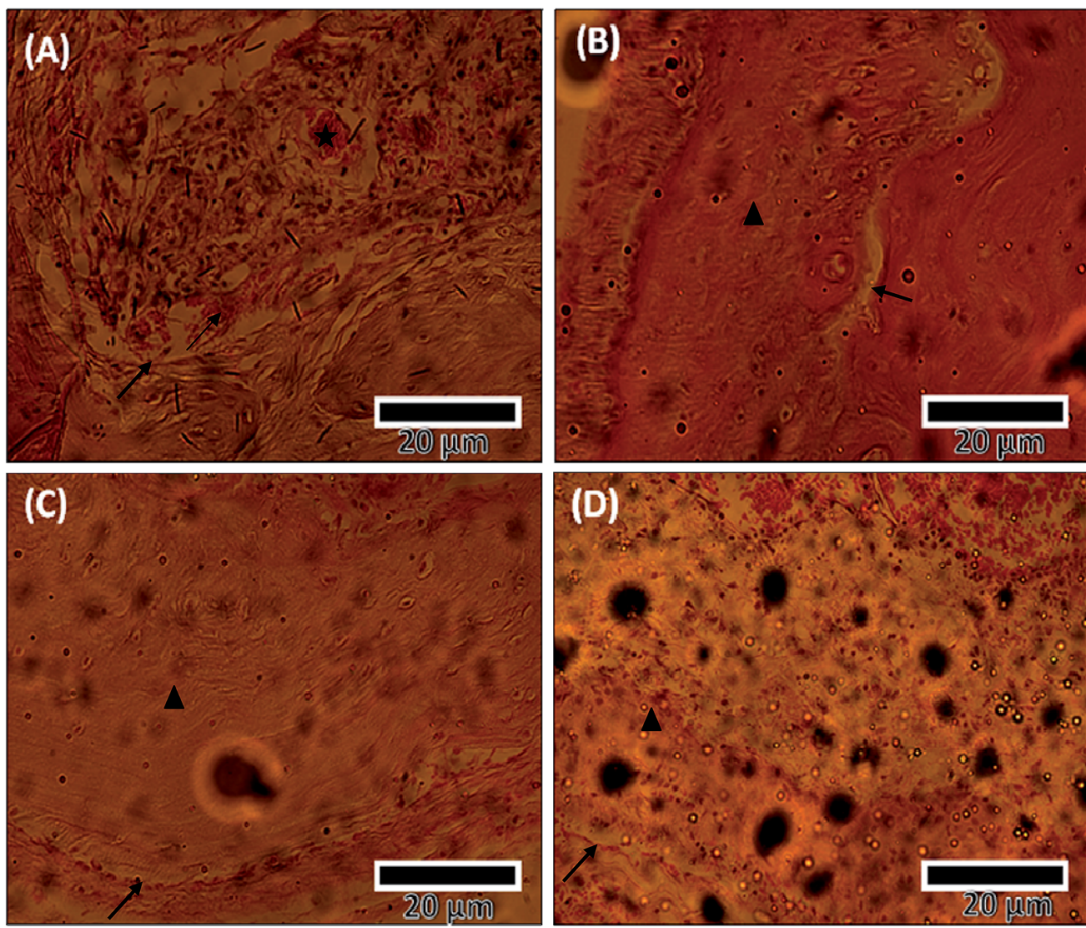

Fig. 8 Histology of treated site and control after 12 weeks using hematoxylin and eosin (H\&E) staining: (A) SHAM; (B) PCL; (C) PCL-HA and (D) $\mathrm{PCL}-\mathrm{HS} \mathrm{PCL}-\mathrm{HA}$ and PCL-HS showed groups higher osteointegration at the defect site than SHAM and PCL groups. $\nearrow$ Defect site boundary, $\star$ inflammatory cells, $\triangle$ neo-bone.

Wistar rats was evaluated after 6 and 12 weeks. These observation periods were chosen according to the degradation profile of the nanofibers in SBF reported earlier. ${ }^{19}$

3.4.2 Organ weight. Organ weight is one of the most sensitive and early drug toxicity indicators, and changes in organ weight often precede morphological changes. It is standard practice to evaluate the effects of drugs or medical devices for toxicology. ${ }^{33}$ After euthanization of the rats, a skin incision was made across the trunk and all the major organs were extracted for gross observation and to compare the change in weight that corresponds to toxicology effect of the composite resorption. Organs that were evaluated were the liver, kidney, heart, testis, epididymis and spleen. While organ weight provides useful signals indicating tested article-related effects, organ weight data are interpreted in an integrated fashion with gross pathology, clinical pathology, and histopathology findings. Detectable weight changes themselves may not necessarily be treatment related or adverse. ${ }^{33}$ There was no significant difference observed among the groups at both time points of 6 and 12 weeks, which indicates no adverse reaction is caused to any organ and therefore we conclude PCL and PCL composites in nanofibrous form are safe (ESI Fig. $3 \dagger$ ). These results agrees with the finding reported when composite of polylactic acid with gelatin and hydroxyapatite was implanted in both male and female rats subcutaneously, no toxic effects were found at the 3 month timepoint. ${ }^{34}$

3.4.3 Hematology. Blood was also extracted to obtain various parameters such white blood cells, red blood cells, hemoglobin, hematocrit, mean cell volume, mean cell hemoglobin, mean corpuscular hemoglobin concentration, red cell distribution width, and platelet count. Toxicology profile of blood did not have any significant difference among groups at both 6 and 12 weeks. Changes in hematology and clinical chemistry parameters provided important information regarding the mechanism of toxicological actions and identification of target tissues with organs. From the diverse nature of hematology parameters that we measured, we briefly provided an overview of the significance of changes in hepatocellular and biliary integrity and function as well as electrolyte balance and blood components. ${ }^{35}$ There was no significant difference seen in any of the other parameters measured though there was difference, though not significant, observed in WBC and hematocrit (Fig. 9); however, these differences were in the mean values, leading to conclusion that there was inflammation at the site because of foreign material present at the site, which ultimately lead to an increase in mean values, keeping other parameters in blood unchanged (ESI Fig. $4 \dagger$ ).

3.4.4 Biochemistry panel of test for toxicity. Serum biochemistry evaluation was performed on an Erba Chem 7 (Erba Mannheim, Germany) semi-auto analyzer using commercial reagent kits. Chemistry panels are groups of tests that are routinely conducted to determine general health status of animals. They help evaluate the body's electrolyte balance and/or the status of several major body organs. The tests were performed on a blood sample drawn from a vein near the eye. We conducted complete serum biochemistry panel test to observe the health of each organ and its functions in all groups. 

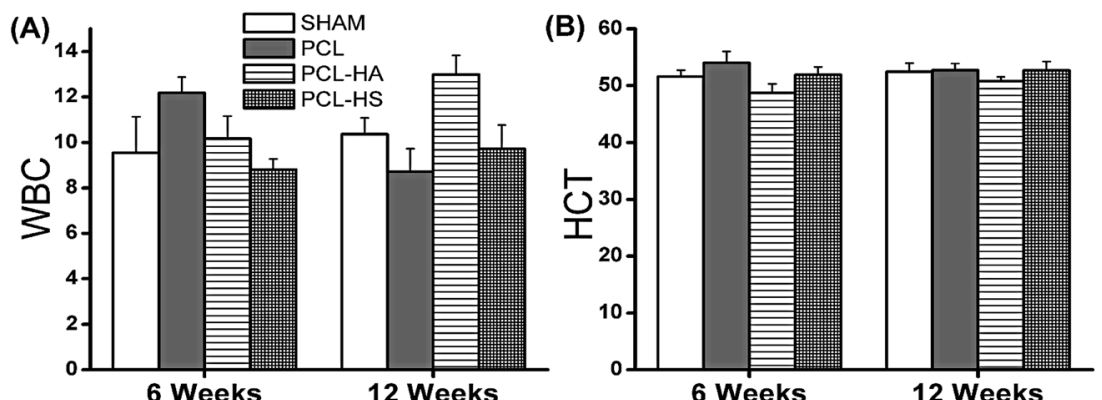

Fig. 9 Difference in mean values with standard error of (A) white blood cells and (B) hematocrit volume observed for all groups at 6 and 12 weeks. There was no significant difference between WBC and HCT among the groups.

The tests were classified under the following panel to study the body chemistry:

Basic metabolic panel (BMP) - usually contains 8 tests, all of which are found in the CMP (below); provides information about the status of rat kidney and respiratory system including electrolyte and acid/base balance with level of blood glucose.

Comprehensive metabolic panel (CMP) - provides the same information as BMP but with addition of the status of a rat's liver with important blood proteins.

Electrolyte panel - detects problem in the rat body's fluid and electrolyte balance (no problems noted in our study).

Lipid profile - assesses a rat's risk of developing cardiovascular disease.

Liver panel (also called hepatic function panel) - screens, detects, evaluates, and monitors acute and chronic liver inflammation (hepatitis), liver disease and/or damage.

Renal profile (also called kidney function panel) - tests albumin, creatinine, BUN, and eGFR for kidney function.

Thyroid function panel - evaluates thyroid gland function (no sign of early thyroid disorders observed in our study).

Elevated creatine level is an indicator of bone mass and quality as shown by various reports that have also reported that creatine monohydrate's role in improving exercise performance and increasing fat-free mass. ${ }^{36}$ There was a significant difference noted in creatine level for the PCL and PCL-HA groups as compared to the PCL-HS and SHAM groups at 6 weeks but not at 12 weeks. Osteoprotegrin (OPG) sysnthesis suppress osteoclast differentiation. Clinical studies shows OPG level in patients with kidney diseases co relate with serum creatinine level. Therefore there may be role of creatine in bone repair. ${ }^{37}$ Earnest et al. conducted a study on creatine supplementation in 34 human subjects and found reduced triglycerides and VLDL cholesterol levels at week four, which were maintained up to week 12, compared to baseline values, showing an inverse dependence between creatine level and triglycerides (Fig. 10). ${ }^{38}$

Triglyceride level in the SHAM and PCL-HS groups was found to be significantly higher than that in the PCL and PCLHA groups at 6 weeks, but at 12 weeks, all groups showed triglyceride formation above the normal range, with no significant difference. This points to better bone healing as increased triglycerides are beneficial, because they promote osteoblastogenesis. Tampakaki et al. studied the role of oleic acid/BSA $(18: 1)$ on osteoclast differentiation and found an increase in intracellular triglyceride accumulation and inhibition of osteoclastogenisis. This establishes that triglyceride formation indirectly affects osteoblastogenesis. ${ }^{39}$ This may lead to the conclusion that SHAM and PCL-HS groups followed different metabolism or cascade of pathway for bone formation compared to the PCL and PCL-HA groups. The other parameters used to check performance of various organs and its functions were found to be in the normal range and no other significant changes were observed (ESI Fig. $5 \dagger$ ).
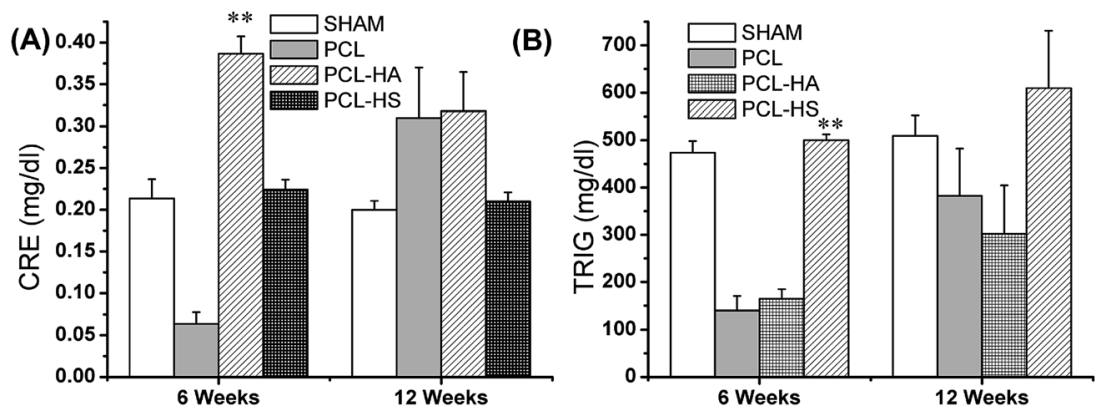

Fig. 10 Elevated values of creatine and triglycerides exhibited by the SHAM, PCL, PCL-HA and PCL-HS groups for bone healing as described in text: $(A)$ creatinine $\left(\mathrm{mg} \mathrm{dl}^{-1}\right)$ and $(B)$ triglycerides $\left(\mathrm{mg} \mathrm{dl}^{-1}\right)$. Significant difference was observed between $\mathrm{PCL}-\mathrm{HA}$ and $\mathrm{PCL}-\mathrm{HS}$ in terms of creatine concentration at the 12 week timepoint. Significant difference was noted in triglyceride concentration between the PCL-HA and PCLHS groups at the 6 week timepoint. 
Table 2 Score and rank for the SHAM, PCL, PCL-HA and PCL-HS groups represented as least + , moderate ++ and most +++

\begin{tabular}{lllll}
\hline Group & SHAM & PCL & PCL-HA & PCL-HS \\
\hline Micro-CT & + & + & ++ & +++ \\
Histology & + & + & ++ & +++ \\
Blood parameter & +++ & +++ & +++ & +++ \\
Serum biochemistry parameter & +++ & ++ & ++ & +++ \\
Overall rank (lower rank is better) & 4 & 3 & 2 & 1
\end{tabular}

\section{Conclusion}

This study highlights the quantitative and qualitative analysis to study the performance and biocompatibility of PCL nanofiber scaffold variations through micro-CT data, histology, hematology and serum biochemistry panel of tests in an $8 \mathrm{~mm}$ critical-sized rat calvarial defect model. It also summarizes qualitative morphological analysis of HA and HS about their crystal structure with polycrystalline phases and their sustained release from the scaffold. Results from this study reveal that all three scaffolds developed and studied here, namely PCL-HS, PCL-HA composites and PCL, are safe for the rat model based on hematology, organ weight and serum biochemistry panel tests. Among the scaffolds, PCL-HS and PCL-HA show significant efficacy to PCL and SHAM control. The PCL-HS and PCLHA groups show comparable performance at 12 week timepoint. The results of the techniques used to measure bone performance among the groups is shown in Table 2.

Our study shows that PCL scaffolds with HA and HS as nanoadditives performed safely, with PCL-HA and PCL-HS both performing superior to PCL alone and the SHAM control. These findings imply that PCL-HS and PCL-HA would be efficient for bone tissue engineering but PCL-HS showed higher and faster bone integration with the adjacent bone at the 6 week timepoint than PCL-HS. Among serum biochemistry parameters, PCL-HS was found to be safer than PCL-HA. Longer-term studies, which would show more complete healing, would provide more insight into their long-term performance in bone tissue engineering. Our study also concluded that HS has potential similar to that HA and it could replace HA as a zinc and silicate containing substitute.

\section{Conflict of interest}

There are no conflict of interests to declare.

\section{Acknowledgements}

This work was supported by IITB Healthcare Consortium and Department of Biotechnology (BT/PR3138/NNT/28/550/2011). Authors also acknowledge Dr B. Ravi and Dr Rupesh Ghyar from OrthoCAD Lab/BETiC for MIMICS ${ }^{8}$; Dr Atul K Singh and Mr Surendra Kumar Verma from Silicate Lab for analysis; and Sophisticated Analytical Instrument Facility (SAIF), Industrial Research and Consultancy Centre (IRCC), Central instrument facility, IIT Bombay, for providing with characterization and testing facilities.

\section{References}

1 R. Vasita and D. S. Katti, Int. J. Nanomed., 2006, 1, 15-30.

2 X. Liu and P. X. Ma, Ann. Biomed. Eng., 2004, 32, 477-486.

3 M. Navarro, A. Michiardi, O. Castaño and J. A. Planell, J. R. Soc., Interface, 2008, 5, 1137-1158.

4 L. Polo-Corrales, M. Latorre-Esteves and J. E. Ramirez-Vick, J. Nanosci. Nanotechnol., 2014, 14, 15-56.

5 J. Lu, M. Descamps, J. Dejou, G. Koubi, P. Hardouin, J. Lemaitre and J.-P. Proust, J. Biomed. Mater. Res., 2002, 63, 408-412.

6 A. K. Jaiswal, H. Chhabra, S. S. Kadam, K. Londhe, V. P. Soni and J. R. Bellare, Mater. Sci. Eng., C, 2013, 33, 2926-2936.

7 L. L. Hench, R. J. Splinter, W. C. Allen and T. K. Greenlee, J. Biomed. Mater. Res., 1971, 5(6), 117-141.

8 S. Xu, K. Lin, Z. Wang, J. Chang, L. Wang, J. Lu and C. Ning, Biomaterials, 2008, 29, 2588-2596.

9 M. Yamaguchi, J. Trace Elem. Exp. Med., 1998, 11, 119-135. 10 Z.-S. Tao, W.-S. Zhou, X.-W. He, W. Liu, B.-L. Bai, Q. Zhou, Z.-L. Huang, K.-k. Tu, H. Li, T. Sun, Y.-X. Lv, W. Cui and L. Yang, Mater. Sci. Eng., C, 2016, 62, 226-232.

11 C. Wu, Y. Ramaswamy, J. Chang, J. Woods, Y. Chen and H. Zreiqat, J. Biomed. Mater. Res., Part B, 2008, 87, 346-353.

12 Y. Ramaswamy, C. Wu, H. Zhou and H. Zreiqat, Acta Biomater., 2008, 4, 1487-1497.

13 P. P. Spicer, J. D. Kretlow, S. Young, J. A. Jansen, F. K. Kasper and A. G. Mikos, Nat. Protoc., 2012, 7, 1918-1929.

14 P. Garcia, T. Histing, J. H. Holstein, M. Klein, M. W. Laschke, R. Matthys, A. Ignatius, B. Wildemann, J. Lienau, A. Peters, B. Willie, G. Duda, L. Claes, T. Pohlemann and M. D. Menger, Eur. Cells Mater., 2013, 26, 1-14.

15 J. P. Schmitz and J. O. Hollinger, Clin. Orthop. Relat. Res., 1986, 205, 299-308.

16 N. Sagar, K. Khanna, V. S. Sardesai, A. K. Singh, M. Temgire, M. P. Kalita, S. S. Kadam, V. P. Soni, D. Bhartiya and J. R. Bellare, Mater. Sci. Eng., C, 2016, 69, 700-714.

17 A. Polini, D. Pisignano, M. Parodi, R. Quarto and S. Scaglione, PLoS One, 2011, 6, e26211.

18 W. Zhang, G. Wang, Y. Liu, X. Zhao, D. Zou, C. Zhu, Y. Jin, Q. Huang, J. Sun, X. Liu, X. Jiang and H. Zreiqat, Biomaterials, 2013, 34, 3184-3195.

19 A. K. Jaiswal, H. Chhabra, S. S. Kadam, K. Londhe, V. P. Soni and J. R. Bellare, Mater. Sci. Eng., C, 2013, 33, 2926-2936.

20 P. Kalia, G. Vizcay-Barrena, J. P. Fan, A. Warley, L. Di Silvio and J. Huang, J. R. Soc., Interface, 2014, 11, 20140004.

21 M. H. Mankani, S. A. Kuznetsov, B. Fowler, A. Kingman and P. Gehron Robey, Biotechnol. Bioeng., 2001, 72, 96-107.

22 K. Garg and G. L. Bowlin, Biomicrofluidics, 2011, 5, 013403.

23 M. Chen, P. K. Patra, S. B. Warner and S. Bhowmick, Tissue Eng., 2007, 13, 579-587.

24 S. E. Noriega, G. I. Hasanova, M. J. Schneider, G. F. Larsen and A. Subramanian, Cells Tissues Organs, 2012, 195, 207221. 
25 M. L. Bouxsein, S. K. Boyd, B. A. Christiansen, R. E. Guldberg, K. J. Jepsen and R. Müller, J. Bone Miner. Res., 2010, 25, 1468-1486.

26 G. Wu, M. Pan, X. Wang, J. Wen, S. Cao, Z. Li, Y. Li, C. Qian, Z. Liu, W. Wu, L. Zhu and J. Guo, Sci. Rep., 2015, 5, 16681.

27 N. Sagar, A. K. Singh, M. K. Temgire, S. Vijayalakshmi, A. Dhawan, A. Kumar, N. Chattopadhyay and J. R. Bellare, RSC Adv., 2016, 6, 93768-93776.

28 K. M. R. Nuss and B. von Rechenberg, Open Orthop. J., 2008, 2, 66-78.

29 C. J. Percival and J. T. Richtsmeier, Dev. Dyn., 2013, 242, 909922.

30 A. S. Mistry, Q. P. Pham, C. Schouten, T. Yeh, E. M. Christenson, A. G. Mikos and J. A. Jansen, J. Biomed. Mater. Res., Part A, 2010, 92, 451-462.

31 H. Develioglu, S. Ü. Saraydin, L. Dupoirieux and Z. D. Sahin, J. Biomed. Mater. Res., Part A, 2007, 80, 505-508.
32 S. R. Gomes, G. Rodrigues, G. G. Martins, M. A. Roberto, M. Mafra, C. M. R. Henriques and J. C. Silva, Mater. Sci. Eng., $C, 2015,46,348-358$.

33 R. S. Sellers, D. Mortan, B. Michael, N. Roome, J. K. Johnson, B. L. Yano, R. Perry and K. Schafer, Toxicol. Pathol., 2007, 35, 751-755.

34 A. K. Jaiswal, R. V. Dhumal, J. R. Bellare and G. R. Vanage, Drug Delivery Transl. Res., 2013, 3, 504-517.

35 V. Ahuja and S. Sharma, J. Appl. Toxicol., 2014, 34, 576-594. 36 R. Cooper, F. Naclerio, J. Allgrove and A. Jimenez, J. Int. Soc. Sports Nutr., 2012, 9, 33.

37 K. Wei, Z. Yin and Y. Xie, J. Nephrol., 2016, 29, 349-357.

38 C. P. Earnest, A. L. Almada and T. L. Mitchell, Clin. Sci., 1996, 91, 113-118.

39 Z. Drosatos-Tampakaki, K. Drosatos, Y. Siegelin, S. Gong, S. Khan, T. Van Dyke, I. J. Goldberg, P. C. Schulze and U. Schulze-Späte, J. Bone Miner. Res., 2014, 29, 1183-1195. 